\title{
Sensing the room: an integrated implementation process to visualize indoor temperature data on floor plans
}

Minkyu Shin, Sang-ik Lee, Hyunsoo Lee and Jin-Kook Lee*

\begin{abstract}
Background: Recently multi-use environmental sensors became affordable and easy to control. This improvement enabled us to measure the broad range of indoor environments by using low-priced and controllable sensor devices. As the data set acquired by widely installed environmental sensors increased significantly, the need for making effective use of the data has become of importance. We developed an integrated visualization mechanism to express larger amounts of sensor data.
\end{abstract}

Method: The mechanism for visualization on floor plan described in this paper consists of the following modules: 1) the sensor module; 2) the data collection module; 3) the data processing module; 4) the spatial data module; 5) the sensor location module; and 6) the data visualization module. A demonstration following the mechanism is introduced in this paper for evaluating the integrated visualization approach. We set up a test-purposed and versatile toolkit that is cheaper, smaller and more controllable than conventional tools. We collect indoor environmental data set composed of sequential numeric data so as to use them as parameters for visualization. We inspected three major issues in the process: 1) indoor temperature data of a specific room collected at a second interval; 2) such a data set can be varied by subdivided spots of interest using multiple toolkits; 3 ) as a result, the collection of data is regarded as one type of parameter for visualization on top of the room's floor plan, e.g. a sudden change of sequential numbers.

Results: Indoor environment such as temperature changes are shown as colors overlapped on the floor plan. In this way it may be easier to understand the state of indoor environment and factors which influence the environment. The floor plan based visualization of an indoor environmental element seems intuitive compared to just listing the numeric information.

Conclusion: This paper introduces and demonstrates an integrated approach for visualizing the indoor environment on a floor plan with an actual test case. Combined with the building model, the visualized data can be used for recognition of the factors affecting to the target environmental element. We expect floor plan-based visualization to be used for decision-making.

Keywords: Sensor data; Data visualization; Floor plan-based visualization; Indoor temperature changes

\section{Background}

People spend most of their daily lives indoors, and this simply explains why indoor temperature is such an important environmental factor (Hyo Joo et al. 2011; Yoon and Spengler 1995). Therefore, controlling comfortable indoor environment is essential for the people to keep their emotional and physical states healthy. An additional

\footnotetext{
* Correspondence: designit@hanyang.ac.kr

Department of Interior Architecture Design, Hanyang University, 222Wangsimni-ro, Seongdong-gu, Seoul 133-791, Republic of Korea
}

benefit is better work productivity (Hee-geon et al. 2013; Moon et al. 2009). Conventionally, to measure and collect the indoor environmental data such as temperature data set used to be inconvenient and expensive due to several reasons (Sik and Bae 2012). The multi-use environmental sensors, however, became affordable and easy to control recently, and this enabled us to measure the broad range of indoor environments by using such low-priced and controllable sensor devices (Yick et al. 2008). However, as the data set acquired by widely installed environmental sensors increased significantly, the need for making 
effective use of the data has become of importance (Jisun 2013; Yongdai and Kwang 2013; Yang Koo and Keun Ho 2008). The more sensor data increases, the more potentiality for managing indoor environment and necessity to manage and represent amount of them grow at the same time. Conventionally, charts are frequently used for representing sensor data (Bo-ram et al. 2012; Chauk and Kyung-Ae 2008; Kwon et al. 2008; Sam-gil et al. 2010). Chart is useful to visualize and compare few sensor data but for expressing a number of sensor data, it is ineffective (Seung-heon et al. 2007) and hard to understand the condition of indoor environment. We, therefore, suggest an integrated mechanism to express such a number of sensor data in an effective way. This paper aims to figure out an integrated approach to visualize such conditional factors using indoor temperature changes. The baseline of this approach is to measure the temperature and analyze given conditions (Seung-Chul et al. 2008). This research aims to develop an integrated visualization mechanism using accumulated data from sensors, rather than the issue for data integrity or the analysis of indoor environment. To verify the feasibility of the proposed mechanism in this paper, consequently, we propose an integrated approach to the visualization of indoor temperature changes using several pragmatically implemented software and hardware modules. This is one of the graphical representations on the existing building geometry using sensor devices and sequential processing mechanism. The entire process from sensing indoor environment to processing and visualizing acquired data can be subdivided into three parts. Each part is composed of submodules. After dealing with the functions of each module inside three parts, this paper shows a demonstration on an actual space. The target environmental element to be visual ized is hourly variation of temperature changes.

\section{Methods}

\section{System architecture}

The system architecture for visualizing the indoor environment on floor plans is composed of three parts, as shown in Figure 1: 1) Input data part, 2) Building model part, and 3) Visualization part. In the building model part, the 2D floor plan or 3D building model is loaded which becomes the background for visualization. Also, all the influence factors of the target environment and the reference point of the installed sensors in the building model are included for more accurate analysis. The input data part deals with the environmental data. In this stage, the environmental data are sensed by installed sensors and collected, retrieved, and processed in order to generate the data to be visualized. The outcome data generated in the Input data part are transferred to an infographic and combined with a loaded building model in the visualization part.

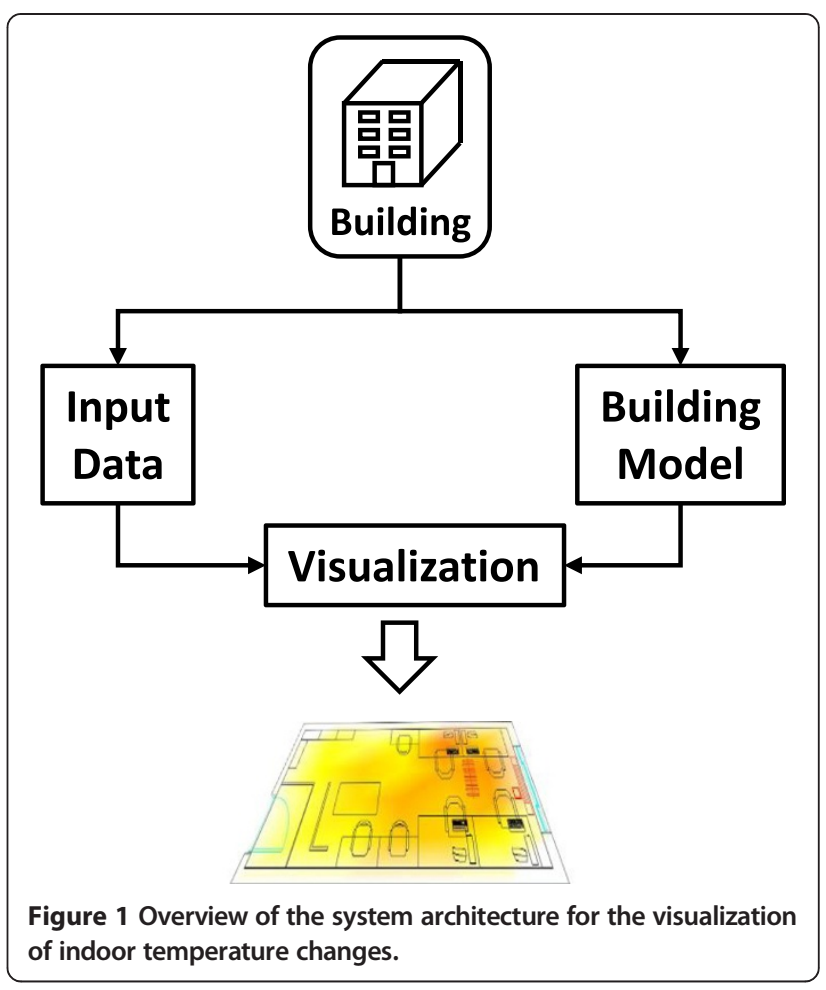

\section{Implementation}

The data flow during the entire visualization process is summarized in Figure 2. Input Data Part is composed of three modules: 1) Sensor Module, 2) Data Collection Module, and 3) Data Processing Module. These three modules measure the target environmental element and process environment raw data. The input data to be visualized generates the outcome data to be visualized. The Building Model Part consists of two modules: 1) Building Model Module, and 2) Sensor Network Module. The Building Model Part loads the building model such as 2D floor plans or 3D building model and determines the reference point of installed sensors. The input data and loaded building model are combined by the Visualization Module in the Visualization Part.

\section{Sensor module}

The Sensor Module measures the target indoor environmental element by multiple sensors and generates sets of raw data. Each sensor senses the numeric value of the target indoor environment at an interval of time, and the data are collected into a set of raw data in the Data Collection Module. The set of raw data is used by visualizing not only condition of indoor environment at realtime but also specific purpose, such as the average value that the user or manager wants to know. Sensing and collecting sufficient number of datasets by installing the appropriate number of sensors is important because the number of input data is closely related with research 


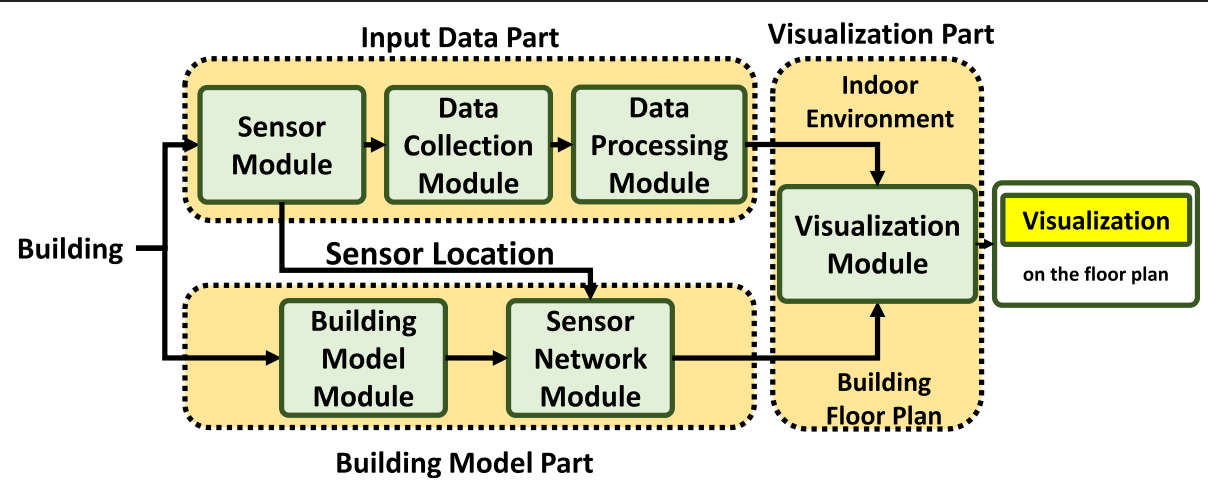

Figure 2 Data flow diagram of the six major modules of implementation.

background and effect of visualization. The greater the input data the more precise and abundant the visualization outcome. As mentioned earlier in scope, the matter of missing or erroneous data will be covered through further work. There are various types of target indoor environments that can be measured such as temperature, humidity, brightness, air quality, and movement. In this paper, the target indoor environment visualized is limited to temperature.

\section{Data collection module}

The Data Collection Module collects the target environmental data measured by the Sensor Module. In this module, the data sensed by the installed sensors each time is accumulated in real-time. The accumulated data is so significant because it enables users to freely modify data according to their intention. This module makes a material difference from conventional monitoring system which can only express environmental data in real-time or a specific moment. Depending on the compatibility between the installed sensors and the visualization software, there are two possible methods to collect data. The two methods are depicted on Figure 3. If the sensors are compatible with Visualization software, the data are able to be collected directly by the Visualization software. Otherwise, if the sensor is incompatible with Visualization software, the collected data should be transferred to a suitable format which is compatible with Visualization software such as csv, DB, or Excel file. If the visualization software is going to be used without function to process accumulated data, user should follow the latter method in order to collect data.

\section{Data processing module}

In order to generate input data, the Data Processing Module retrieves a particular data among the collected raw dataset and calculates the retrieved data according to the purpose of visualization. Defining the input data should be done before selecting a particular part of the data. The target and algorithm for the data processing are decided by the definition of the input data. As in selecting a particular part of the data from a dataset of
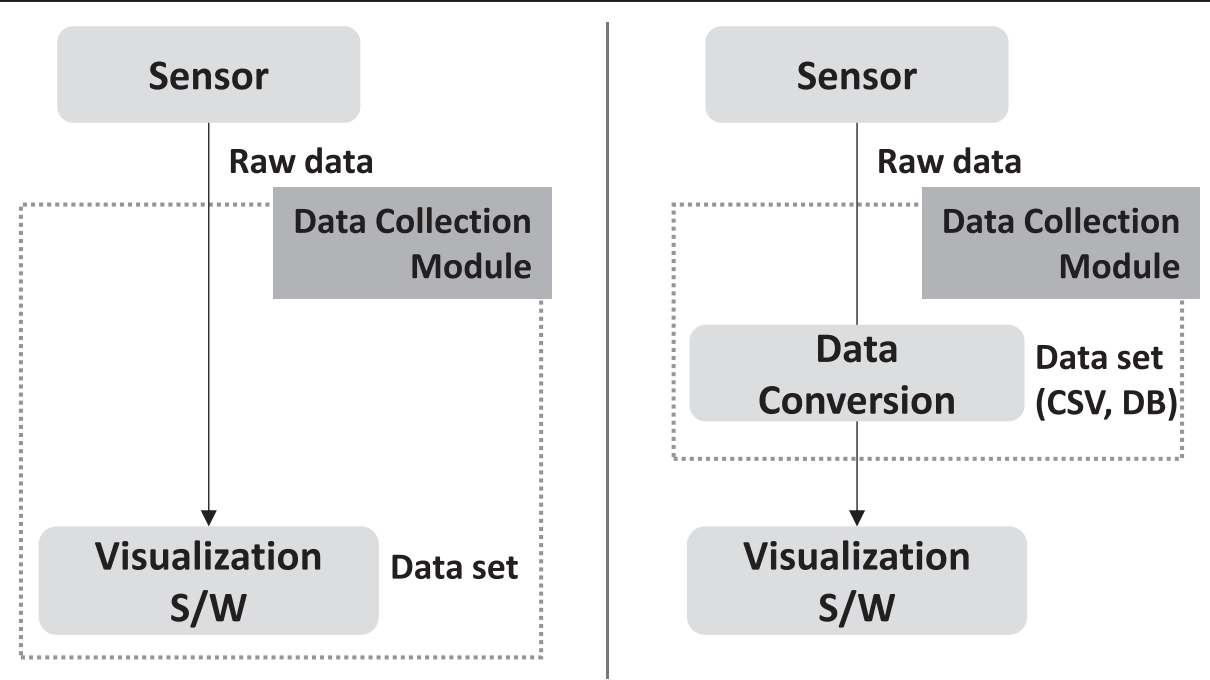

Figure 3 The role of data collection module: without data conversion (left) and with data conversion (right). 


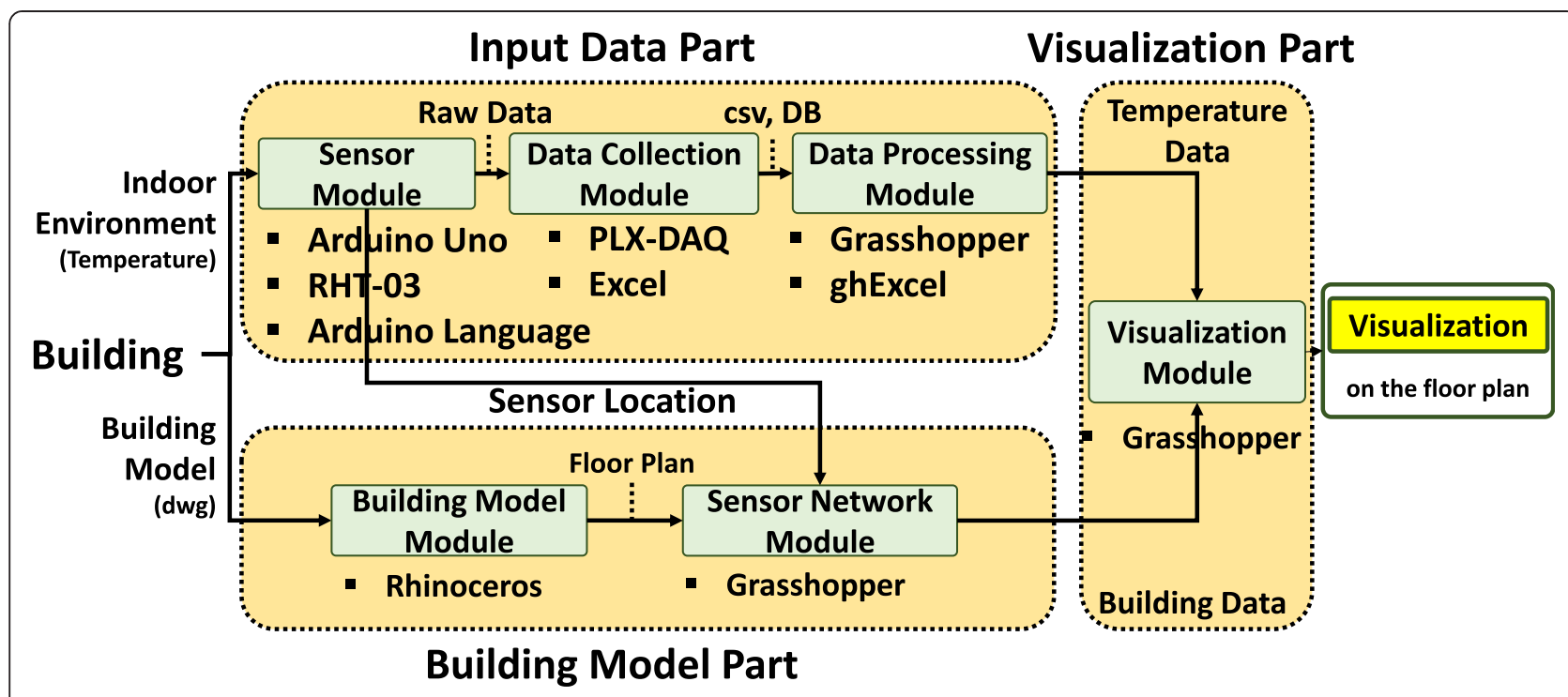

Figure 4 The data flow and software/hardware used in each module.

measured environmental data, it is also possible to decide what environmental element is visualized when there are plural indoor environmental elements collected by the installed sensors.

\section{Building model module}

The Building Model Module loads the building geometry, which becomes the back-layer of the visualization. The loaded building model is used as two ways. One is the reference for designating the boundary of the sensor network layer and another is the data for analyzing the visualization outcome. The building model should meet three requirements: 1) drawing a floor plan with precise scale and shape of the indoor space where sensors are installed; 2) including all the factors that influence the target indoor environment within the space; and 3) designating the sensor location on the floor plan accurately. The use of loaded building model regarding designation of the boundary of the sensor network layer is described on the Sensor Network Module. When the building model is combined with the visualization outcome, the building model becomes the basis of analysis of the visualized indoor environmental data.

\section{Sensor network module}

The Sensor Network Module generates the sensor network layer which becomes the basis for visualization on building model. Based on the loaded building model, the shape and the size of the boundaries of the sensor network layer are defined. The generated sensor network layer is visualized in Visualization Module and mapped with the input data processed by Data Processing Module. Each sensor location, which is mapped with the input data, can be acquired by Indoor Positioning System (IPS).
There are several possible IPS systems such as using Wi-Fi, Global Positioning System antenna, or Geo-Magnetism. Without IPS, the sensor location can be designated manually comparing given building model with the sensor location in actual space. The process of making sensor network layer consists of two steps: 1) defining the boundary of sensor network layer by designating edge of floor plan; and 2) designating the sensor points on the boundary.

\section{Visualization module}

Visualization Module transforms the numeric input data into visual information using sensor network layer. Each input data of an installed sensor is connected to each sensor point on the sensor network layer. Connected with the input data, the sensor points move along the z-axis. As the sensor points move depending on input data, sensor network is transformed accordingly. The transformed sensor network enables input data to be expressed in any type

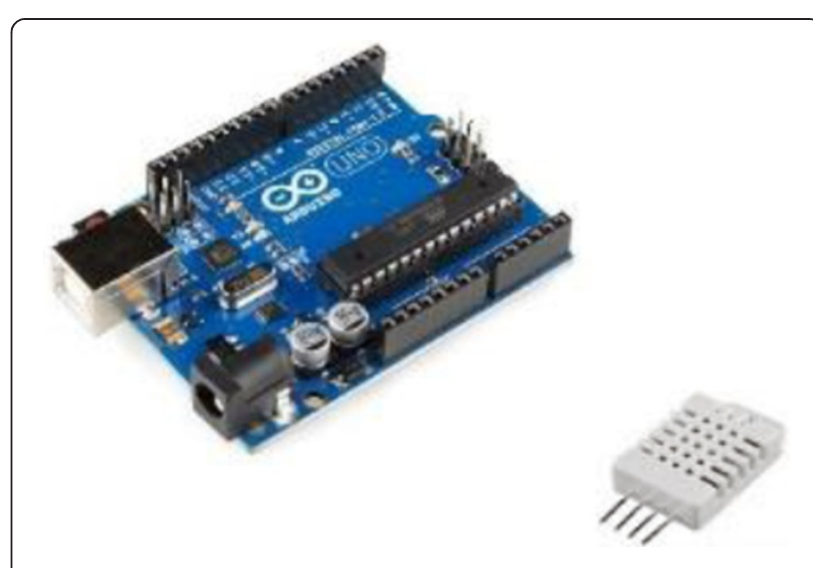

Figure 5 Arduino Uno Board (left) and RHT03 sensor (right). 


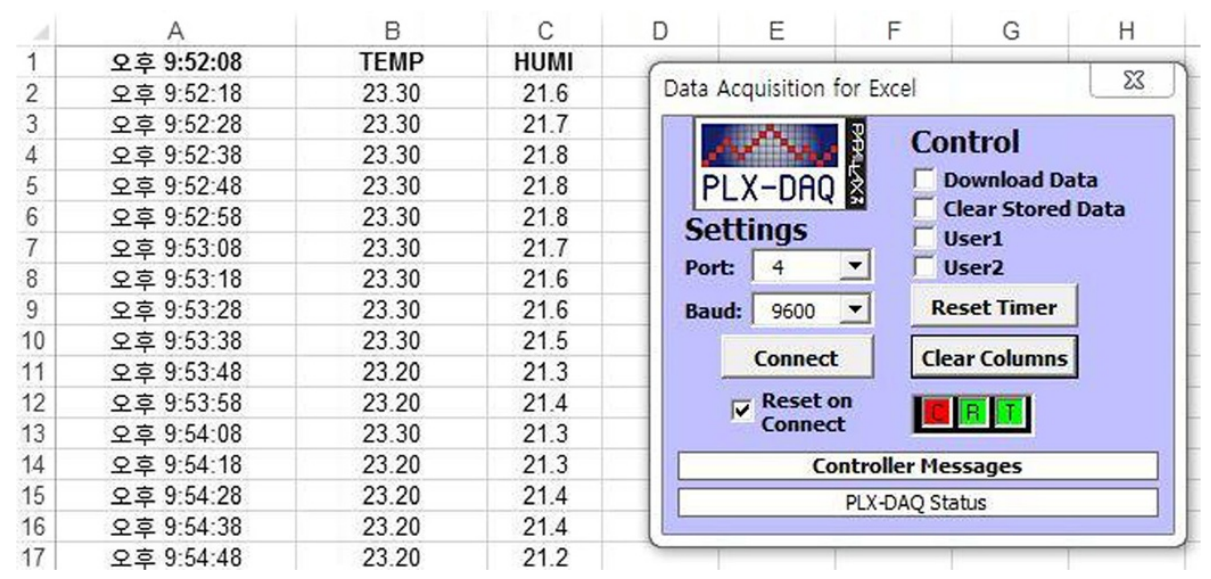

Figure 6 Exporting the measured data into Excel spreadsheet file using PLX-DAQ plug-in.

of graphical image including color or geometry. In this step, the visualization method is decided by the building model and the definition of the input data, considering how to visualize the interested environmental data in the most intuitive way. When the visualization method is decided and the input data is connected to each sensor point, the sensor network layer will be transformed into a certain form of visual information, according to the decided visualization method and the processed input data.

\section{Demonstration}

To examine feasibility of proposed mechanism We demonstrated an integrated approach for visualizing the changes of indoor temperature in the laboratory room in which the researchers are working. For this demonstration, we installed 12 sensors on the ceiling by considering how people use this room. This room is located in building of College of Human Ecology, Hanyang University. Figure 4 represents the overall flow of the demonstration including the software/hardware used in each stage.

\section{Measurement of temperature}

Arduino Uno Board and RHT03 sensors shown in Figure 5 were used as instruments measuring the temperature, which are cheap and easy to control. RHT03 is a pennysized temperature/humidity sensor that is able to measure from -40 to 80 degrees $C$ temperature range and also has $+/-2 \%$ error range in humidity (Maxdetect). Arduino

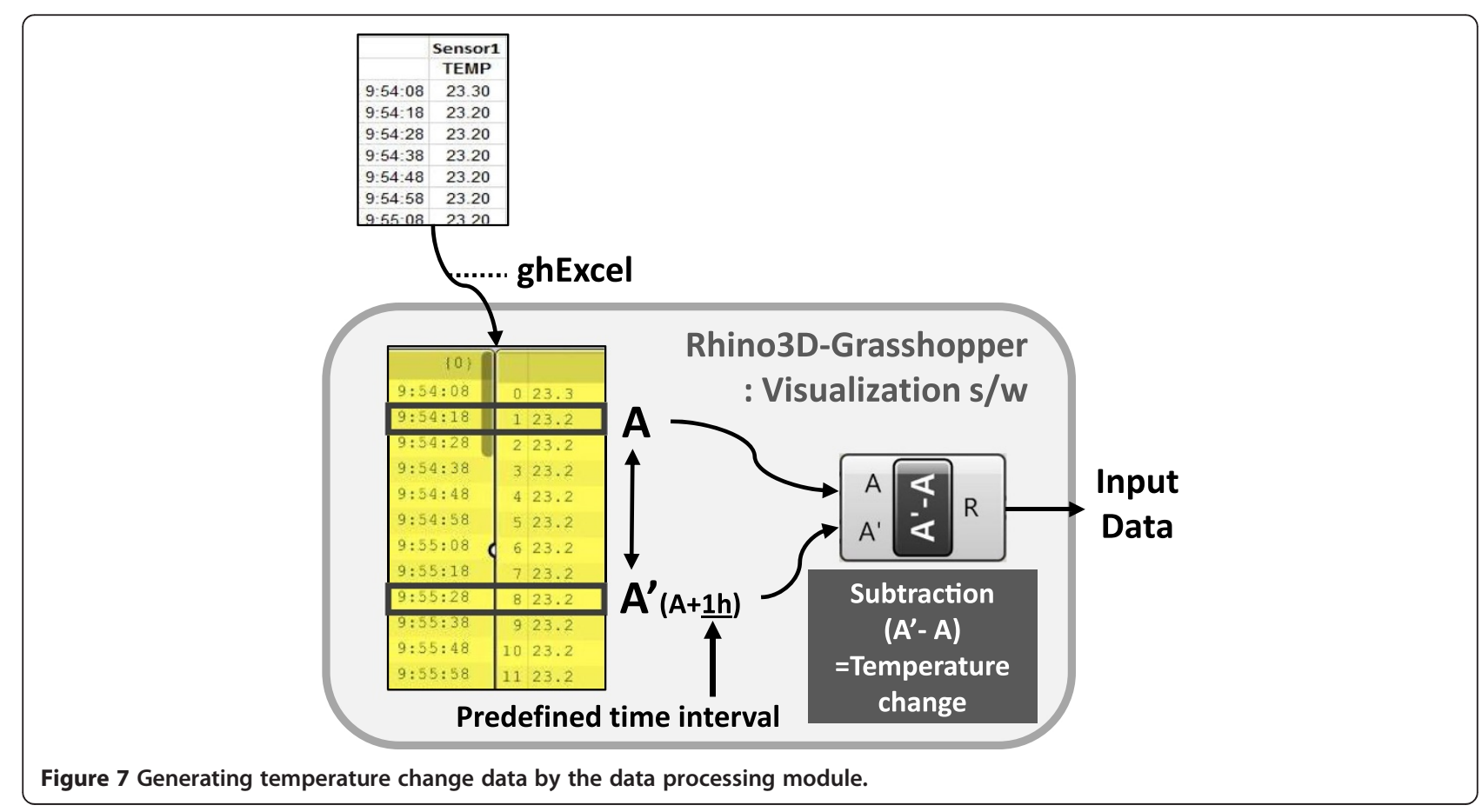




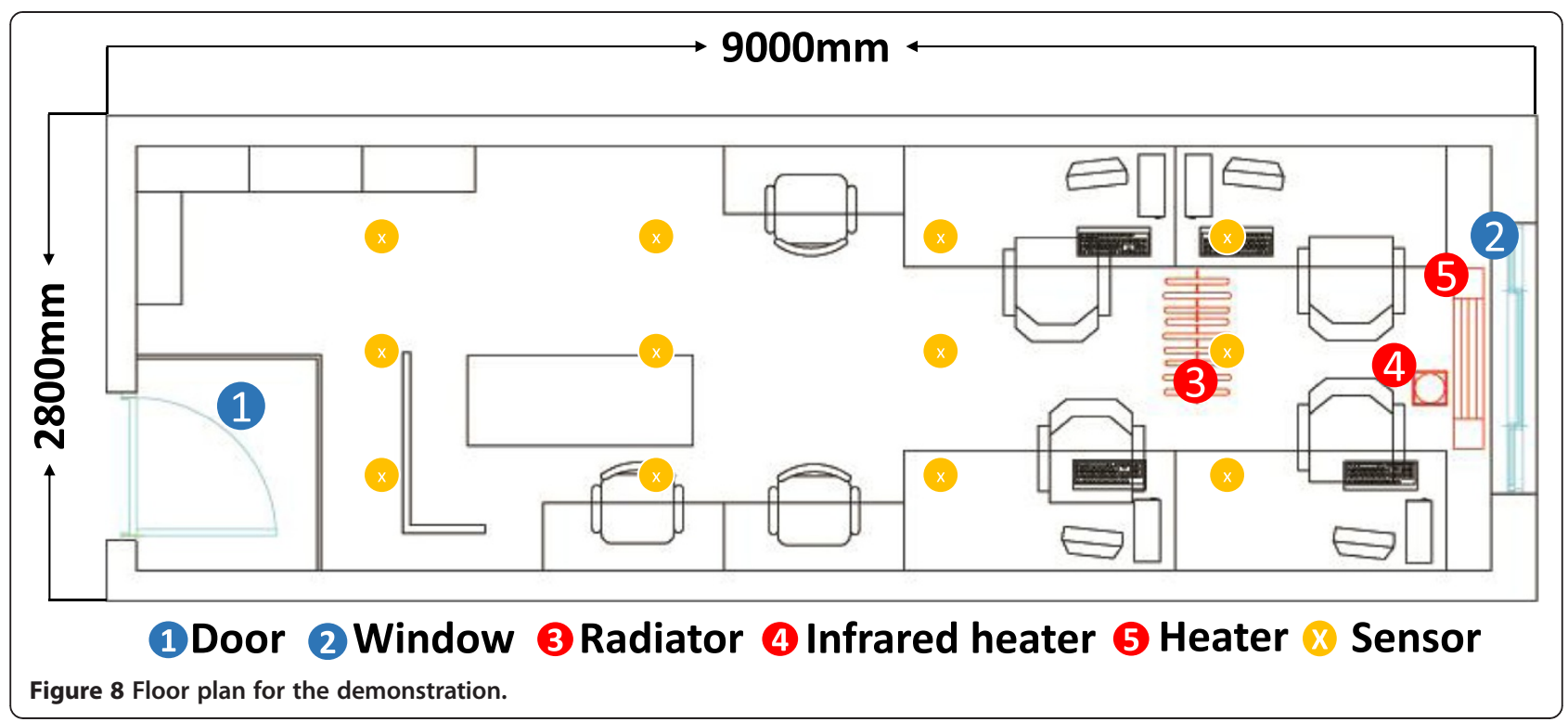

Uno Board is a microcontroller board controlled by Arduino Language (Arduino). Using the language, it is possible to control how the sensors operate and in what form the data will be collected. We easily revised source code for operating RHT03 by using Arduino Integrated Development Environment which is provided from the website for free because almost all the source codes are provided by manufacturer. The revised source code was compiled and uploaded from computer to Uno board through USB. In this paper, the sensors operated with 60 second intervals and temperature was collected as degrees Celsius.

\section{Converting and collecting the measured data}

The measured environmental data was collected in an Excel spreadsheet due to the issue of compatibility with Rhino3D-Grasshopper (Rhinoceros; Grasshopper) on which the environmental data will be visualized. As illustrated in Figure 6, an Excel plug-in software named PLX-DAQ (Parallax Data Acquisition tool) was used for exporting the measured data from the Sensor Module into an Excel file (Parallax). We use PLX-DAQ because this software and manuals are offered free on the websites. Users just download and follow the manuals for operating this software. Each dataset measured by the sensors is collected in each spreadsheet and a dataset includes temperature, humidity and the time the sensor operated. The collected dataset was used for multipurpose like calculating the average or subtracting between specific times in Processing Module.

\section{Temperature change data processing}

The process from data processing to visualization were implemented using Rhino3D-Grasshopper in this demo, as described in Figure 7. The Grasshopper is compatible with Excel spreadsheet file using a plug-in program named ghExcel (Xiaoming). The datasets consist of time, humidity and temperature values in 1 minute intervals. The target environmental element is temperature. In order to generate the final input data from the collected datasets, the input data needs to be defined before selecting a particular part of data. In this demo, we defined the input data as the temperature changes at hourly intervals. That means the subtraction of two temperature values between two moments at an hour interval. As one interested moment is selected, the temperature values of the selected moment and 1 hour later are retrieved. After retrieving two temperature values to be compared, the temperature difference is generated for the final input

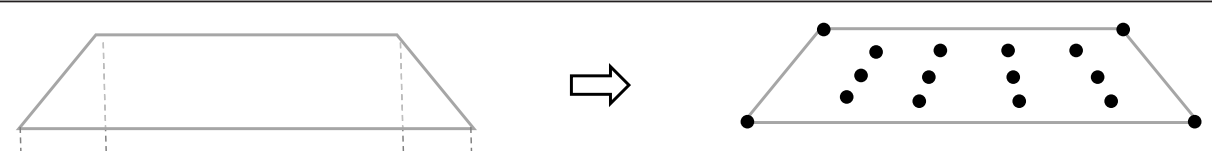

Figure 9 Generation of sensor network layer. 

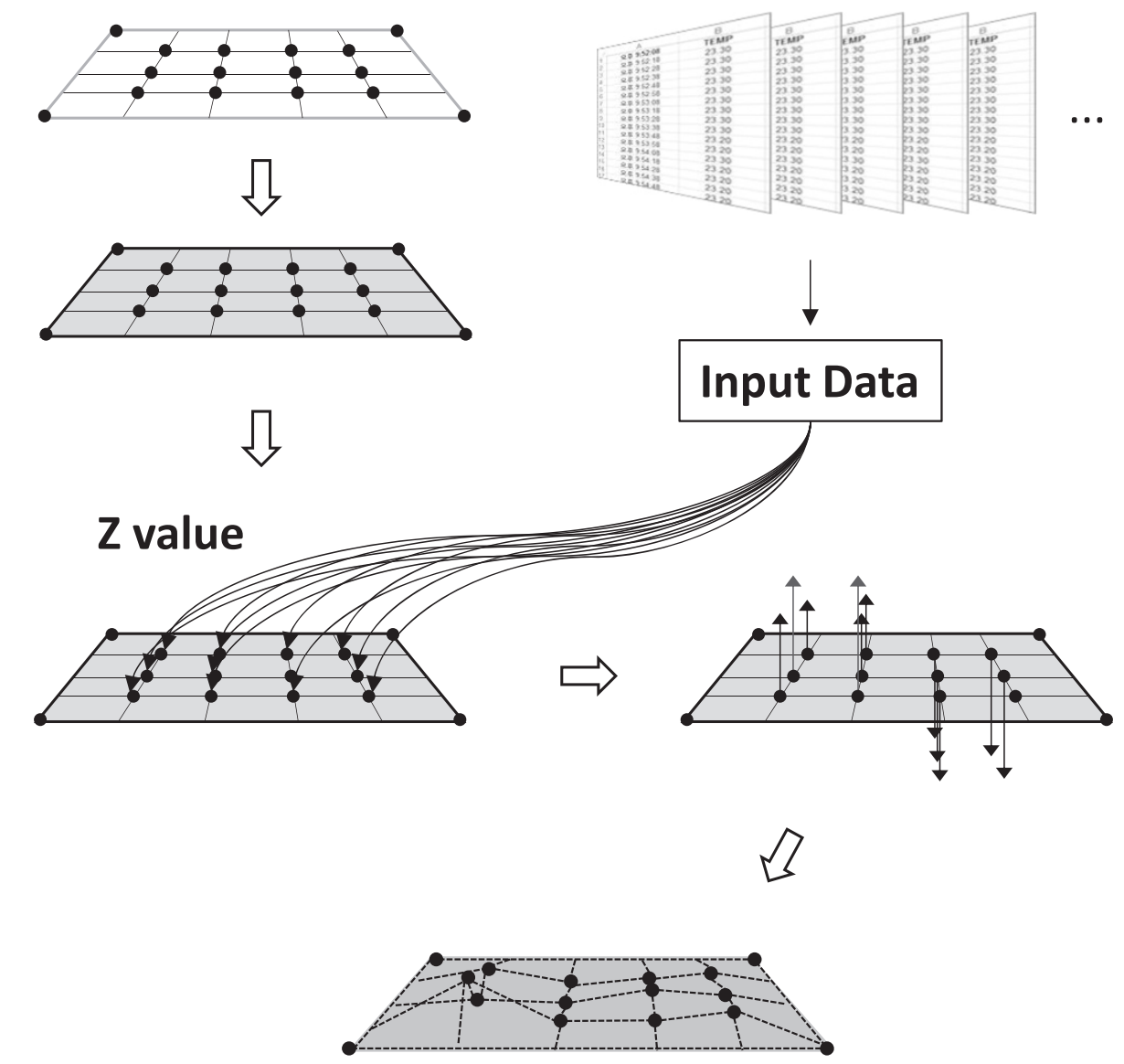

Figure 10 Process of generating geometry using grid segments.

data. This demo used Rhino3D-Grasshopper "slider" function for selecting interface for retrieval.

\section{Importing floor plan}

As the building model for this demo, the floor plan was imported to Rhino3D. The size and layout of the space are shown in Figure 8. In addition, sensors used in this demonstration and all the building elements affecting temperature such as heaters or openings are expressed too. Those elements are crucially used in analysis for visualization outcome.

\section{Generating sensor network layer}

Before the visualization stage, sensor network layer was made above the floor plan. The sensor network layer becomes the basis when transferring the input data into

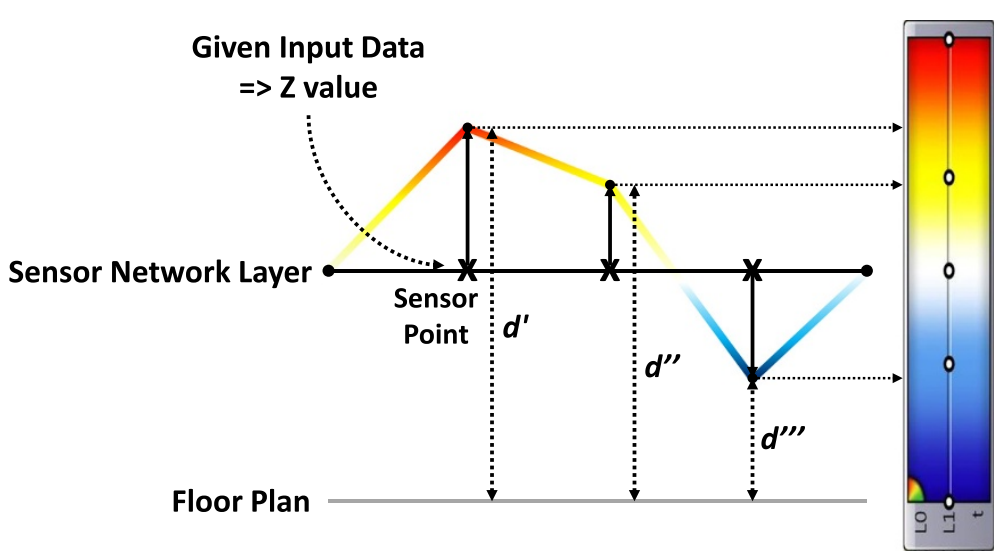

Figure 11 Visualization mechanism of converting $z$ value to color gradation. 
visual information. The series of process to generate sensor network are represented in Figure 9. Pointing at the corners of the floor plan's outline, the boundary of sensor network layer was defined. On the boundary of layer, each sensor point was created on each sensor location. The sensor points were designated on the layer manually based on the comparison of floor plan with the actual space, because IPS, by which the sensor location is acquired, is not used in this demo.

\section{Visualizing input data}

For the visualization of the given input data above the floor plan, the sensor network layer is divided into grid segments with each sensor point as the center. The

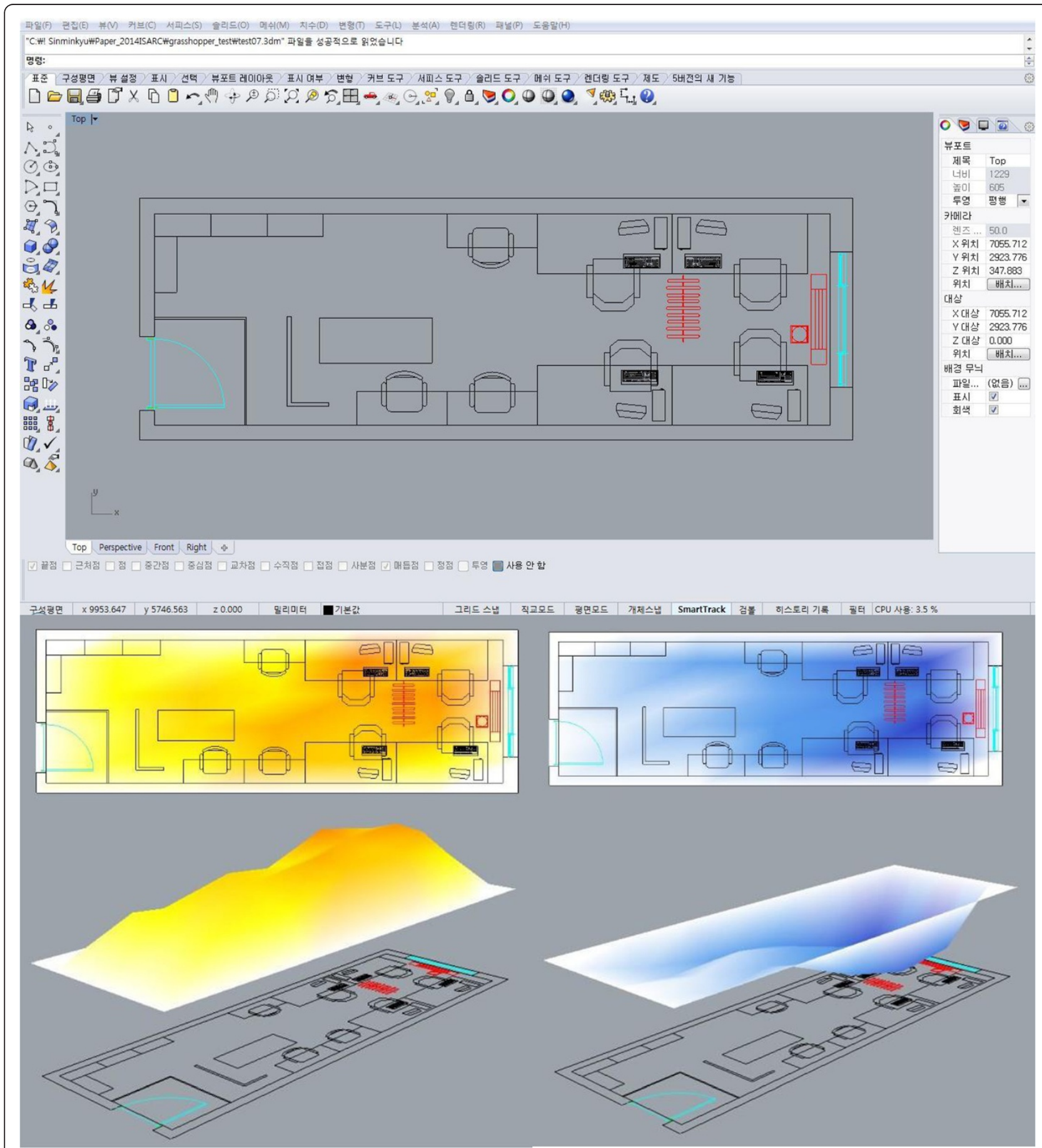

Figure 12 Result of temperature changes visualization. 
divided layer forms a flat surface which is able to be connected with the input data on each sensor point. The given input data are assigned to each sensor point as $\mathrm{z}$ value of Rhino3D. As the sensor points move to the $\mathrm{z}$ axis direction, the surface is transformed in accordance with the sensor points moving into an uneven surface. The entire process of transforming sensor network layer is illustrated in Figure 10. At the same time, it is possible to express the temperature change as color as well as the geometry.

As shown in Figure 11, according to the distance to the floor plan, the surface is colored with red-to-blue color gradation. As a result, input data assigned as $\mathrm{z}$ value are transformed into visual information such as geometry and color.

\section{Results and discussion}

Figure 12 shows the final visualization outcome on Rhino3D viewport. In top view, the temperature changes are shown as colors overlapped on the floor plan. In this way it is easier to realize the state of indoor environment and factors which influence the temperature changes. In isometric view, the degree of temperature changes appears in the form of geometry which looks like a colored uneven plane. The three-dimensional visualization of an indoor environmental element is intuitive compared to two-dimensional or numeric information. The input data of the left visualization result is the temperature changes between 9 am - 10 am when researchers went to lab and turned on the heaters. The outcome shows that the overall temperature was raised centrally around the heater. In contrast, the input data of right visualization result is the temperature changes between $9 \mathrm{pm} 10 \mathrm{pm}$ when researchers got off and opened window intentionally in order to make drastically temperature changes. As a result, the temperature decreased rapidly during 1 hour with the heater and window as the center because the heater was turned off and the window was opened.

\section{Conclusions}

This paper introduces and demonstrates an integrated approach for visualizing the indoor environment on a floor plan with an actual test case. The entire system consists of three major parts, which are able to be subdivided into 6 modules. Several prerequisites exist in each module such as precise floor plan. As shown in Section 3 Demonstration, which is visualizing temperature changes in a position for an hour, visualization mechanism proposed in this paper is able to process the numeric data and to convert processed data into visualized data. As a result, we find out that the visualized environmental data are more intuitive and easier to figure out the state of an indoor environment compared to numeric data. Combined with the building model, the visualized data can be used for recognition of the factors affecting the target environmental element. This information enables floor plan-based visualization to be used for improved decision-making. When changing the layout of an existing building, we can use not virtual data acquired by simulation but also the visualized data acquired by real sensor as evidence for design decision-making. In terms of monitoring, intuitive visualized data can help people evacuate under emergency, showing where an accident happened. Applicability can be expanded as the number of type of sensor. To visualize not only temperature changes but also other indoor environment, we need to develop the visualization mechanism, considering various kinds of available sensors and their network and visualization methods. We will continue to enhance the capability of the data visualization mechanism depicted in this paper on a given building model, based on various types of available sensor modules, their network, or alternated data collection.

\section{Competing interests}

The authors declare that they have no competing interests.

\section{Authors' contributions}

$J \mathrm{~L}$ defined the topic and scope of the work, directed the entire processes of this study. MS and SL co-developed the visualization process, conducted the experiment and drafted the manuscript. HL supported the experiment. All authors read and approved the final manuscript.

\section{Acknowledgement}

This research was supported by a grant (13AUDP-B068892-01) from Architecture \& Urban Development Research Program funded by Ministry of Land, Infrastructure and Transport of Korean government.

Received: 30 June 2014 Accepted: 24 October 2014

Published online: 12 November 2014

\section{References}

Arduino. Arduino Uno board. In http://arduino.cc/en/Main/ArduinoBoardUno. Accessed 10 June 2014.

Bo-ram, K, Soo-Hyun, P, \& Bong-Hee, H. (2012). Indoor environment monitoring system. Proceedings of the KIISE Korea Computer Congress, 39(1B), 57-59.

Chauk, K, \& Kyung-Ae, C. (2008). Implementation of a monitoring software using environment sensor data. Proceedings of the KIISE Korea Computer Congress, 35(2B), 309-313.

Grasshopper. Grasshopper. In http://www.grasshopper3d.com. Accessed 10 June 2014. Hee-geon, P, Myeong-jin, K, \& Yong-shik, K. (2013). Measurement and assessment of indoor air quality in newly built university buildings. Journal of the Korean Society of Living Environment System, 20(2), 216-224.

Hyo Joo, K, Geun Young, Y, \& Jeong, K. (2011). A field survey of thermal comfort in office building with thermal environment standard. Journal of the Korea Institute of Ecological Architecture and Environment, 11(3), 37-42.

Jang Sik, B. (2012). Efficient energy management utilizing prediction model in the sensor network based environment monitoring system. Yeungnam University: Ph.D Dissertation.

Jisun, L. (2013). A Study on visualizing method and expression of information design for big data. Korean Society of Basic Design and Art, 14(3), 261-269.

Kwon, S, Park, D, Cho, Y, Park, E, Kim, S, \& Jeong, M. (2008). Temperature and humidity monitoring using Ubiquitous Sensor Network (USN). In Proceeding of the 46th Meeting of KOSAE Korean Society for Atmospheric Environment (pp. 457-458).

Maxdetect. RHT03 Digital Humidity \& Temperature Sensor. In http://www.humiditycn.com/cp22.html. Accessed 10 June 2014.

Moon, S-W, Kim, T-W, Jang, D-S, \& Hong, W-H. (2009). A study on occupants' subjective evaluation and a measurement of indoor environments of an office building. Journal of the Architectural Institute of Korea, 25(5), 279-286. Parallax. PLX-DAQ Data Acquisition for Excel. In https://classic.parallax.com/tabid/ 393/Default.aspx. Accessed 10 June 2014. 
Rhinoceros. Rhino3D. In http://www.rhino3d.com. Accessed 10 June 2014. Sam-gil, C, Ki-tael, K, \& Dong-il, K. (2010). A study of environment monitoring system based on USN. Journal of Information and Convergence Communication Engineering, 14(6), 1488-1492.

Seung-Chul, L, Sang-Joong, J, Young-Dong, L, \& Wan-Young, C. (2008). Design and implementation of flooding-based query model in wireless sensor networks for indoor environmental monitoring system. Journal of the Korean Sensors Society, 17(3), 168-177.

Seung-heon, S, Kyeong-jin, B, \& Eung-kon, K. (2007). A study on visualization technology for environmental sensor data. Proceedings of Korea Institute of Information and Communication Engineering, 1, 192-197.

Xiaoming. GhExcel - Interface with Excel. In http://www.food4rhino.com/project/ ghexcel. Accessed 10 June 2014.

Yang Koo, L, \& Keun Ho, R. (2008). Historical sensor data management using temporal information. Journal of Korea Spatial Information Society, 10(4), 97-102.

Yick, J, Mukherjee, B, \& Ghosal, D. (2008). Wireless sensor network survey. Elsevier Computer Networks, 52, 2292-2330.

Yongdai, K, \& Kwang, H, C. (2013). Big data and statistics. Journal of the Korean Data Information Science Society, 24(5), 959-974.

Yoon, D-w, \& Spengler, JD. (1995). Standards for indoor air pollutant levels and ventilation rates. Journal of the Architectural Institute of Korea Special Issue, 39(6), 12-17.

doi:10.1186/s40327-014-0010-2

Cite this article as: Shin et al:: Sensing the room: an integrated

implementation process to visualize indoor temperature data on floor plans. Visualization in Engineering 2014 2:10.

\section{Submit your manuscript to a SpringerOpen ${ }^{\circ}$ journal and benefit from:}

- Convenient online submission

- Rigorous peer review

- Immediate publication on acceptance

- Open access: articles freely available online

- High visibility within the field

- Retaining the copyright to your article

Submit your next manuscript at $\gg$ springeropen.com 